\title{
Fluorescence Optical Imaging of Juvenile Arthritis
}

STEPHANIE GABRIELE WERNER, MD; HANS-ECKHARD LANGER, MD, RHIO Rheumatology, Immunology, Osteology Center Düsseldorf, Düsseldorf; GERD HORNEFF, MD, Professor of Medicine, Department of Pediatrics, Asklepios Clinic St. Augustin, Sankt Augustin, Germany. Address correspondence to Dr. Werner; E-mail: s.werner@rheuma-online.de. J Rheumatol 2011;38:1447; doi: 10.3899/jrheum.101351

In juvenile idiopathic arthritis (JIA), valid detection of involved joints is essential for correct classification, therapeutic decisions, and prognosis ${ }^{1}$. Clinical assessment is a particular challenge, and reliable results depend on the ages of the children and their ability to cooperate. Ultrasonography (US) and magnetic res-

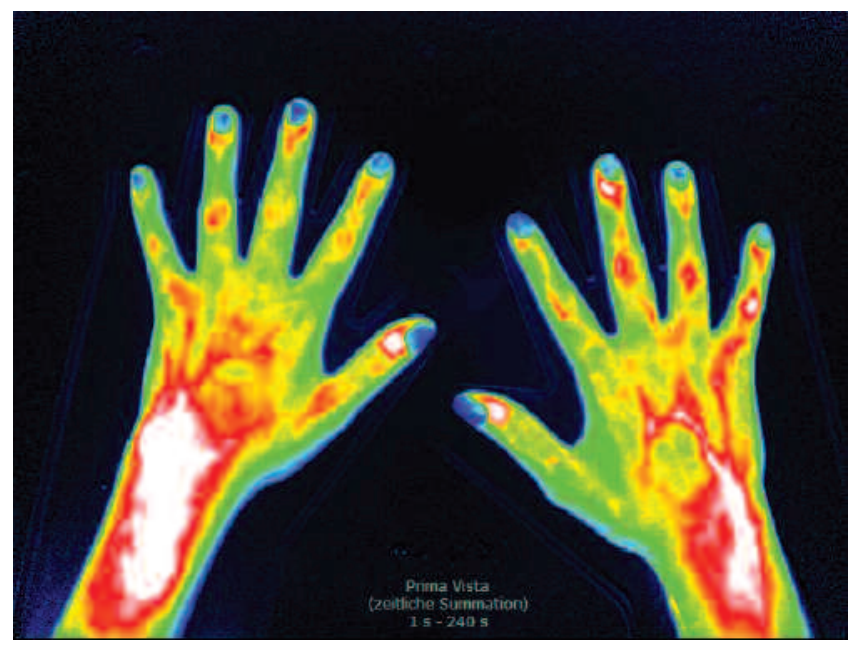

Figure 1. Fluorescence optical imaging (FOI) of the hands of a 15-year-old girl with juvenile idiopathic arthritis and disease recurrence after treatment reduction. Ultrasonography with power Doppler signals revealed swelling and exudation of the metacarpophalangeal (MCP) joints and synovitis of the wrists. FOI showed increased signal intensities (white and red areas) at the inflamed nailbeds and in the region of proximal interphalangeal joints, MCP joints, and wrists.

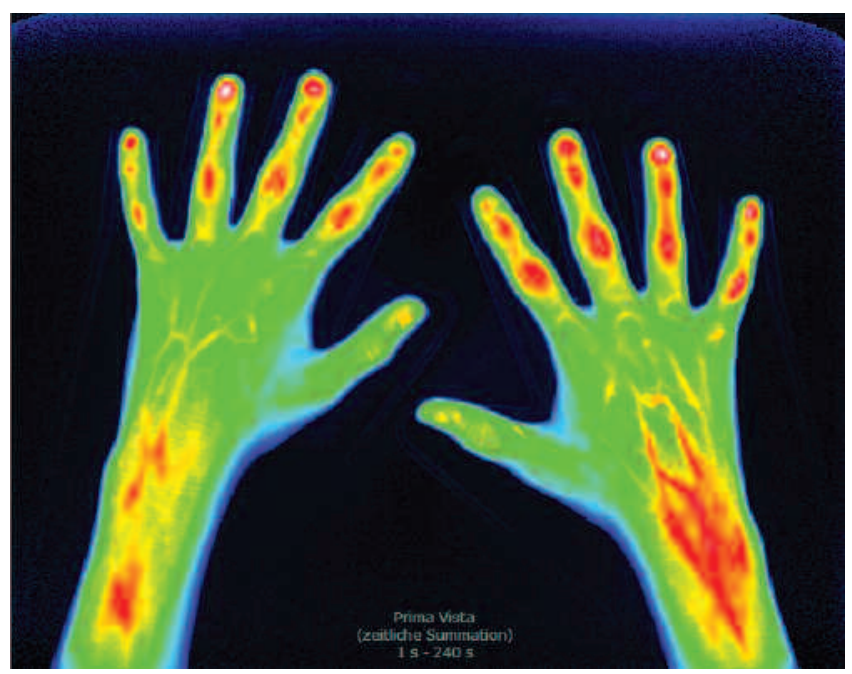

Figure 2. Fluorescence optical imaging (FOI) of the hands of a 15-year-old girl with very early juvenile idiopathic arthritis. Ultrasonography revealed exudation of proximal interphalangeal (PIP) joints. FOI displayed mainly symmetric increased signal intensities in the projection of all PIP and distal interphalangeal joints and both wrists.

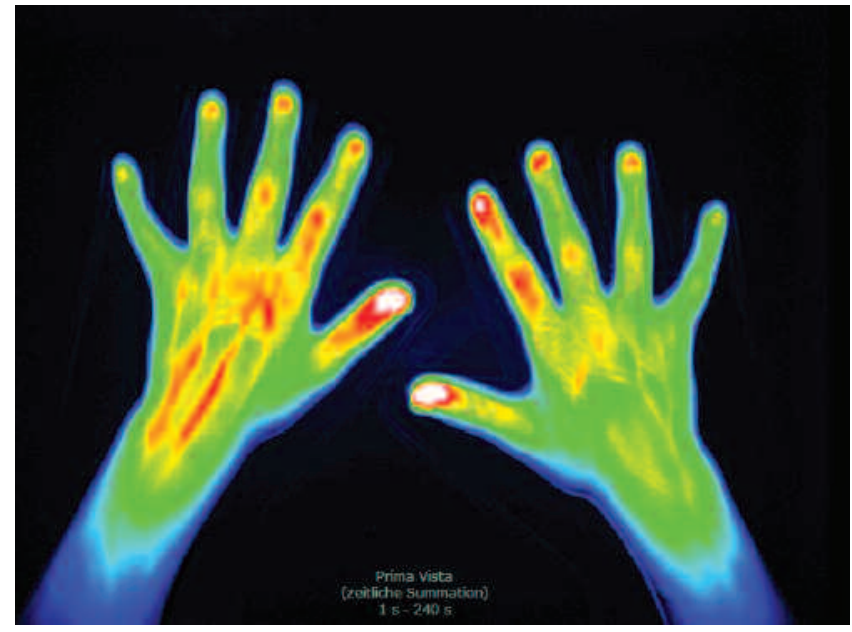

Figure 3. Fluorescence optical imaging (FOI) of a 13-year-old girl who was initially diagnosed with oligoarticular juvenile psoriatic arthritis. FOI displayed focal increased signal intensities in numerous finger joints and resulted in an altered diagnosis of polyarthritis.

onance imaging are used for imaging in JIA, but have practical limitations. Fluorescence optical imaging (FOI) enables visualization of inflammation in arthritis and related conditions ${ }^{2,3}$. We report our first experiences with FOI in children with arthritis using the Xiralite-System (mivenion $\mathrm{GmbH}$, Berlin, Germany) with indocyanine green as a fluorophor $(0.1 \mathrm{mg} / \mathrm{kg} / \mathrm{BW}$ intravenous; Pulsion Medical Systems, Munich, Germany).

In 2 children, FOI corresponded well with the clinical and US findings (Figures 1 and 2). The third child complained of symptoms in numerous joints, but was diagnosed with oligoarthritis after clinical and US examination. FOI displayed polyarticular involvement (Figure 3) and resulted in initiation of methotrexate treatment, according to national guidelines ${ }^{1}$.

FOI might be a new tool for assessment of active disease in JIA. FOI can offer the needed advancement in assessing polyarticular disease in JIA, with the goal of targeted therapy and improved treatment outcome.

\section{REFERENCES}

1. Guellac N, Niehues T. Interdisciplinary and evidence-based treatment guideline for juvenile idiopathic arthritis. Klin Padiatr 2008;220:392-402.

2. Bremer C, Werner S, Langer HE. Assessing activity of rheumatic arthritis with fluorescence optical imaging. Eur Musculoskelet Rev 2009;4:96-100.

3. Fischer T, Ebert B, Voigt J, Macdonald R, Schneider U, Thomas A, et al. Detection of rheumatoid arthritis using non-specific contrast enhanced fluorescence imaging. Acad Radiol 2010;17:375-81. 